\title{
Research On Sun Shadow Positioning Genetic Algorithm Based On BP Neural Network
}

\author{
Xu Ying \\ North China Electric Power University in Baoding, Hebei, China \\ 2536145609@qq.com
}

\begin{abstract}
Keywords: Fitting shadow length, Least square fitting, Genetic algorithm based on BP neural network, Global search, Simulated annealing
\end{abstract}

\begin{abstract}
In this paper, the model of the solar shadow end plane coordinates and the measurement of the latitude and longitude, and the measuring time are established. The solution of the video shooting location and date is given by the change of the length of the shadow in the video. And the application of genetic algorithm based on BP neural network to the model error test and evaluation. If there were the date and time in the video, then the angle could be obtained, and the declination angle would be found directly by the relationship between the latitude angle and the dates with the known to rod length. Then because the ratio of $\tan \partial$ is equal to the length of the rod and the shadow length, we can find out high noon sun angle and obtain the latitude and longitude after the fitting of the short film, corresponding to the calculated the noon solar altitude angle. When there was no date and time in the video, then according to the data of the solar shadow vertex coordinates of a fixed straight bar on the horizontal ground, the most suitable place and date of the straight rod is determined. This is an optimization problem, combined with global search and simulated annealing, two methods are listed to solve the objective function.
\end{abstract}

\section{Introduction}

How to determine the video was filmed on location and shooting date is an important aspect of video data analysis, sun shadow positioning technology is through solar shadow variation of objects in video analysis, a method to determine the video was filmed on the place and date.

\section{The Establishment and Solution of the Model}

The Mathematical Model of the Change of the Shadow Length. On earth a point by changing sunlight conditions, is by the earth itself and the rotation of the earth around the sun causes, the sun shadow positioning technology need to accurately grasp the mathematical model of the variation of the shadow and the shadow length changes, known of the sun's shadow length and measurement of sun's altitude, latitude and longitude location factors relationship, to quantitative analysis of these parameters for objects with long shadow effects, the introduction of common physical[1][3]: declination angle said Sun location coordinates and the earth connection and equatorial plane angle, azimuth said coordinates of the location where the sun in the equatorial plane of the projection of straight line and the direction of the north angle, as shown in Fig.1.

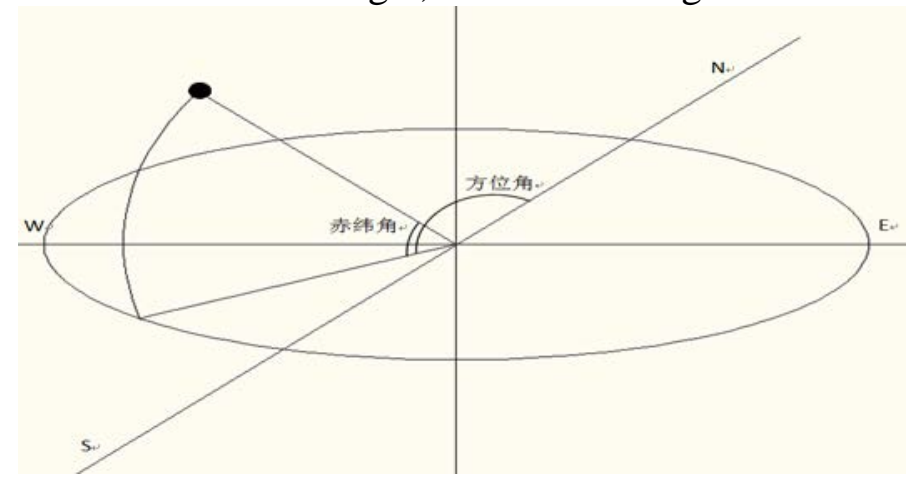

Fig 1 coordinates of the location 
In addition, effect of sun shadow length of the most direct factor for solar altitude angle, namely directed towards the object of sun light and object the angle between the latitude and longitude of the ground. As shown in Fig.2, the higher the sun is, the greater the solar zenith angle between the sun and the earth.

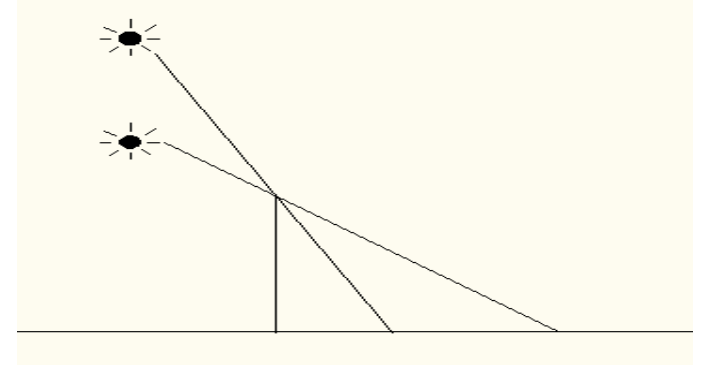

Fig 2 the solar zenith angle

In the calculation of the length of the solar shadow, taking into account the influence of the earth rotation, the data show that the rotation of the earth every hour of the angle of rotation, the angle of the expression is:

$$
\begin{aligned}
& \sigma=15 t \\
& t=m-12
\end{aligned}
$$

Represents 24 hours of time, for the time period, the value of the $\theta$ [5]. The expression of the:

$$
\begin{aligned}
\theta & =\left[\begin{array}{l}
0.006918-0.399912 \cos (b)+0.070257 \sin (b)-0.006758 \cos (2 b) \\
+0.000907 \sin (2 b)-0.002697 \cos (3 b)+0.00148 \sin (3 b)
\end{array}\right] \\
b & =\frac{2 \pi(N-1)}{365}
\end{aligned}
$$

The expression of the solar altitude angle [4] is:

$$
\partial=\arcsin \{\sin (\beta) \times \sin (\theta)+\cos (\theta) \times \cos (\beta) \times \cos (\sigma)\}
$$

To stand on the ground of wood at the bottom as the origin of coordinates, the ground plane, then coordinate the shadow endpoint was 0 . Assuming that the coordinates of the shadow endpoint, then the following distance is calculated:

$$
\begin{aligned}
& L=O P^{\prime}=\sqrt{\left(x_{0}-0\right)^{2}+\left(y_{0}-0\right)^{2}} \\
& \frac{L}{H}=\cot \partial \\
& H \cot \partial=\sqrt{x_{0}^{2}+y_{0}^{2}}
\end{aligned}
$$

$y_{0}=\tan \beta \times x_{0}$

The above part of the symbol is as follows:

$m$ - Time under 24 hours

$t$ - Time under 12 hours

$p$ '- Projection point at the end of the shadow 
$N$ - Number of days from January 1st of this year to the date of survey

$H$ - Stick height

$L$ - Stick shadow

The sun shadow end coordinate model obtained according to the change of the period value which is shown as Table 1, is follows as (11). The data curve of the sun shadow length is shown as Fig.3.

Table 1 shadow length of the different period

\begin{tabular}{llllll}
\hline \multicolumn{5}{c}{ shadow length [m] } \\
\hline 6.65 & 6.12 & 5.66 & 5.27 & 4.94 & 4.65 \\
4.39 & 4.18 & 3.99 & 3.83 & 3.69 & 3.57 \\
3.48 & 3.41 & 3.35 & 3.32 & 3.3 & 3.31 \\
3.33 & 3.37 & 3.43 & 3.51 & 3.61 & 3.73 \\
3.88 & 4.05 & 4.25 & 4.47 & 4.74 & 5.04 \\
\hline
\end{tabular}

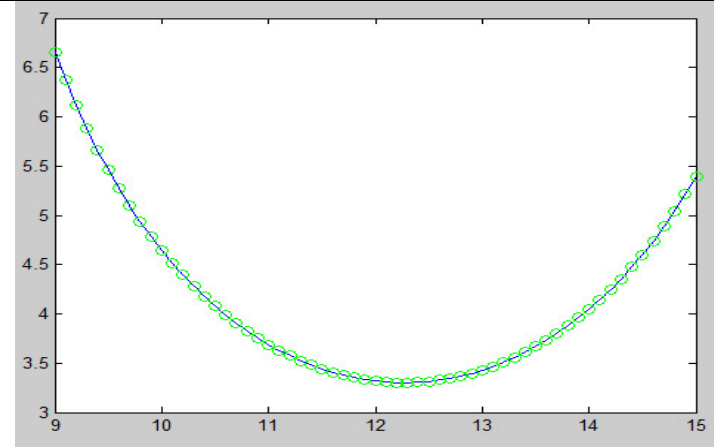

Fig.3 the data curve of the sun shadow length

$y=0.101 x^{3}+0.653 x^{2}-11.42 x+63.7$

\section{According to the Shadow Coordinates to Determine the Location and Time}

Determine the Longitude. First in Appendix 1 to the data fitting, the selection of Lagrange interpolation polynomial fitting. Because the Lagrange interpolation method to fit the curve has high smoothness, continuous curvature.

The basis functions of Lagrange interpolation are:

$$
\begin{aligned}
& l_{i}(x)=\frac{\left(x-x_{0}\right) \mathrm{L}\left(x-x_{i-1}\right)\left(x-x_{i+1}\right) \mathrm{L}\left(x-x_{n}\right)}{\left(x_{i}-x_{0}\right) \mathrm{L}\left(x_{i}-x_{i-1}\right)\left(x_{i}-x_{i+1}\right) \mathrm{L}\left(x_{i}-x_{n}\right)}=\prod_{\substack{j=0 \\
j \neq i}}^{n} \frac{x-x_{i}}{x_{i}-x_{j}}, i=0,1,2, \mathrm{~L}, n . \\
& L_{n}(x)=\sum_{i=0}^{n} y_{i} l_{i}(x)=\sum_{i=0}^{n} y_{i}\left(\prod_{\substack{j=0 \\
j \neq 0}}^{n} \frac{x-x_{j}}{x_{i}-x_{j}}\right)
\end{aligned}
$$

\section{$l_{i}(x)$-Lagrange Ki function \\ $L_{n}(x)$-Lagrange interpolation function}

Determine the Latitude. By (8) transformation, the relationship between the length of the rod and shadow length can be obtained, that long rod and the length ratio of the solar altitude angle of the cotangent value. Fitting using the same ideas and requirements of solar altitude angle of the optimal solution, you need to fit between the elevation angle of the sun and shadow track, so you can assume that the pole length is 3 meters, using polynomial method on the elevation angle of the 
sun and shadow length to fit the shadow to take the shortest point corresponding to the noon solar altitude angle.

Fitting function

$$
y=0.0028 x^{2}-0.2680 x+6.9765
$$

Solution $\quad \partial=87.6^{\circ}$

According to the solar altitude angle is optimized at noon[5][2], can be obtained by measuring point latitude, expression is as follows: The height of the sun angle optimization is $\partial=67.4140^{\circ}$, there are

$$
\phi=\left(90^{\circ}-|\partial \pm \lambda|\right)
$$

Extract Video Image Length Information. Extracting gray images with MATLAB, as shown in Fig.4.

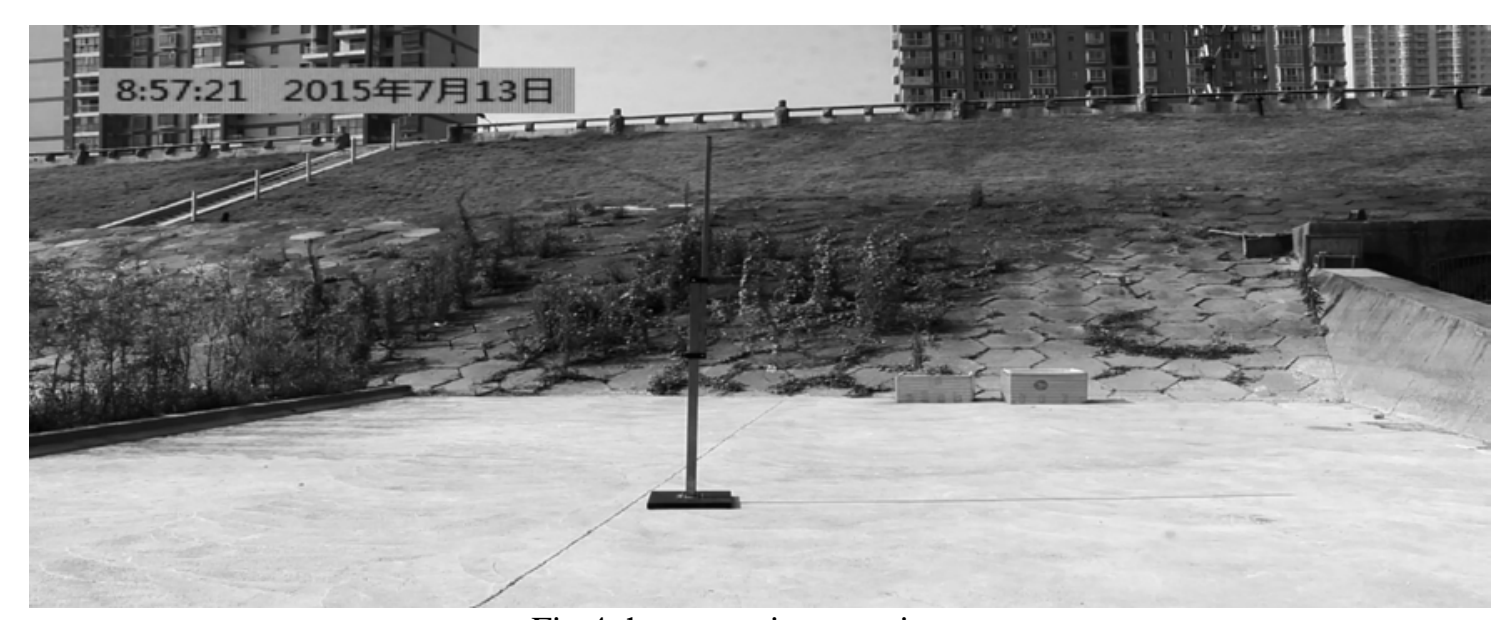

Fig.4 the extracting gray image

The corresponding different time shadow length values are as follows as Table 2.

Table 2 the relationship between time and shadow length

\begin{tabular}{cc}
\hline time $[\mathrm{s}]$ & shadow length $[\mathrm{m}]$ \\
\hline 8.9616 & 15.8 \\
8.9719 & 15.9 \\
8.975 & 15.8 \\
8.9766 & 15.7 \\
8.9858 & 15.7 \\
8.9913 & 15.8 \\
8.9938 & 15.8 \\
8.9983 & 15.7 \\
9 & 15.7 \\
9.0128 & 15.7 \\
\hline
\end{tabular}

Draw the data in the table into a fitting function to get the results shown as Fi.5. 


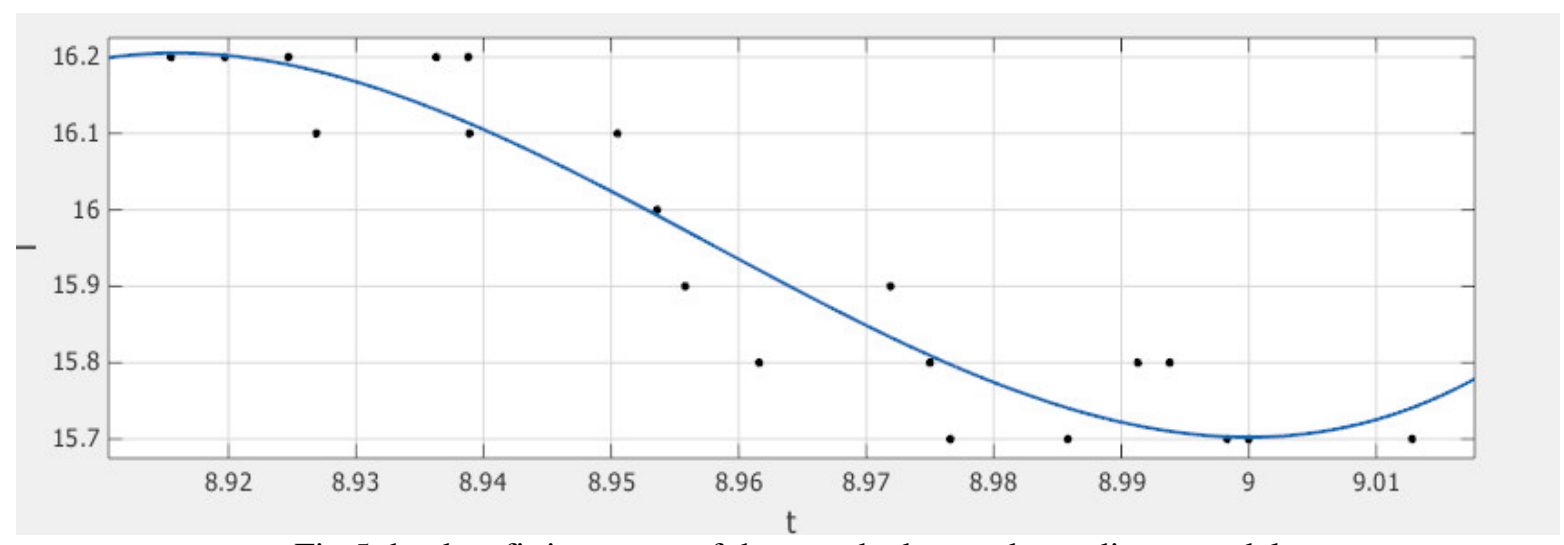

Fig.5 the data fitting curve of the sun shadow end coordinate model

According to the turning point from the video picked the shadow along with the time variation of the data and the data by fitting the income to image function, still can like the first few asked as to find the image, in fitting the image to determine the inflection point is, then convert the longitude. According to image fitting to obtain the shortest shadow long is 15.8 meters, because of the long rod has been given, solar altitude angle tangent value is two constant shadow long and rod length ratio. Ask the sun elevation angle and then seek latitude, and the first two questions of the process, similar, here not to do a detailed description, the place is obtained:

In the video didn't give a date and time, or county from the video data interception and fitting and follow-up with simulated annealing algorithm for solving optimal latitude, with the third asked the same, final results for 54 degrees north latitude, longitude 109.1877. The point in the territory of Mongolia.

\section{Summary}

The whole paper is based on the basic knowledge of astronomy and geography, which is not included in the consideration of the factors such as the refraction of the atmosphere, the surface angle of the sun, the height of the height of the sun, and the height of the sun.

Research model is ideally the altitude of the sun and shadow length changes. As a matter of fact, the obliquity of the ecliptic inner bias force for solving and shadow length change path and time, the shadow length change path and geographical coordinates of the concrete function has certain influence on the latitude and longitude.

\section{References}

[1] Jiang Qiyuan. Mathematical model (Third Edition). Beijing: Higher Education Press ,2003.

[2] Han G. Mathematical modeling method and its application. Beijing: Higher Education Press, 2005.

[3] Wu Lin. Research on the latitude and longitude estimation based on the shadow of the sun. Journal of Tianjin University, 2010, 12 (1): 27-28.

[4] Zheng Pengfei, Lin Dajun, Liu Xiaoyang. Research on technology of shadow line return lighting effect based on mechanical engineering and power engineering, East China University of Science and Technology, 2002,37.

[5] Wang Heping. The shadow inquiry trajectory. China teaching monthly, 2010 (9): 29-30. 